\title{
Is quadriceps H-reflex excitability a risk factor for patellofemoral pain?
}

\author{
Marcella Ferraz Pazzinatto ${ }^{\mathrm{a}, *}$, Danilo de Oliveira Silva ${ }^{\mathrm{a}, \mathrm{b}}$, Evangelos Pappas ${ }^{\mathrm{c}}$, \\ Fernando Henrique Magalhães ${ }^{\mathrm{d}}$, Fábio Mícolis de Azevedo ${ }^{\mathrm{a}}$ \\ a University of São Paulo State, School of Science and Technology, Physical Therapy Department, Presidente Prudente, Brazil \\ b La Trobe Sports and Exercise Medicine Research Centre, School of Allied Health, La Trobe University, Bundoora, Victoria, Australia \\ c The University of Sydney, Faculty of Health Sciences, Sydney, Australia \\ d University of Sao Paulo, School of Arts, Sciences, and Humanities, Sao Paulo, Brazil
}

\begin{abstract}
A B S T R A C T
Patellofemoral pain (PFP) is one of the most common conditions in orthopedic practice while recent evidence has suggested that it may be a predisposing factor to patellofemoral osteoarthritis. In addition to biomechanical alterations associated with the pathomechanisms underlying PFP, the investigation of neurophysiological alterations has provided novel information in the understanding of the pathophysiology of PFP. For instance, women with PFP present lower amplitude of the vastus medialis (VM) H-reflexes compared to pain-free controls, which suggests that the excitability of spinal reflexes might be a promising tool for discriminating woman with PFP in clinical practice. However, the cross-sectional design of the current research does not inform whether the reduced excitability predisposes to or is the consequence of PFP. Therefore, two hypotheses can be raised: (1) the reduction in excitability of the $\alpha$-motoneurons is a risk factor for PFP; Or, (2) the reduction in H-reflex excitability is a consequence of PFP due to pain. If the former hypothesis is proven correct, it may help in the early identification of individuals with PFP. If the latter, it may help understand the reduced excitability as a consequence of the long-term pain, which may be interfering in the recovery of individuals with PFP in a long-term basis. In addition, exploring such hypotheses may have direct rehabilitative and prevention implications for PFP and its putative progression to knee osteoarthritis.
\end{abstract}

\section{Introduction}

Knee disorders are common causes of physical disabilities that affect millions of people around the world [1]. Among a large variety of knee impairments, patellofemoral pain (PFP) has identified as one of the most common condition in orthopedic practice [2]. PFP is defined as pain around or behind the patella, which is aggravated by activities that load the patellofemoral joint (PFJ) during weight bearing on a flexed knee, such as stair negotiation and running [3]. The prevalence of PFP in women aged between 18 and 35 years is 13\% [4]. Moreover, the incidence rate is higher in women than in men, with women 2.23 times more likely to develop PFP [5] Recent evidence has suggested that PFP may be a predisposing factor to patellofemoral osteoarthritis [6,7], which is associated with a high economic and clinical burden, in terms of both direct and indirect health-related costs $[8,9]$.

A variety of biomechanical alterations (e.g. at the hip, knee and ankle levels) have been associated with the pathomechanisms underlying PFP [10-13]. However, evidence-based treatments are not effective in the long-term, with more than $50 \%$ patients reporting continuing symptoms after treatment [14]. This finding indicates that the mechanisms behind PFP are currently not fully understood and/or are not being properly addressed [14].

The investigation of neurophysiological alterations associated with PFP have provided novel information in order to understand the pathophysiology of PFP [15-17]. For instance, De Oliveira Silva et al. [16] reported that the amplitude of the vastus medialis (VM) H-reflexes are lower in women with PFP as compared to pain-free controls. The authors speculated that the excitability of spinal reflexes might be a promising tool for discriminating women with PFP in clinical practice [16]. However, the cross-sectional design of this research does not inform whether the reduced excitability is related with the development of PFP or simply its consequence. Therefore, the hypothesis is that the reduction in the excitability of the monosynaptic spinal reflex of the quadriceps may be a risk factor of PFP. If the hypothesis is proven correct, it may help in the early identification of individuals with PFP. If not, it may help understand the reduced excitability as a consequence of the long-term pain, which may be interfering with the recovery of individuals with PFP in a long-term basis. In addition, exploring such a

\footnotetext{
* Corresponding author at: Department of Physiotherapy FCT/UNESP, Roberto Simonsen Street, 305, CEP 19060-900, Presidente Prudente (SP), Brazil.

E-mail address: ferraz_mar@hotmail.com (M.F. Pazzinatto).
} 
hypothesis may lay the foundations of rehabilitative and prevention strategies designed to avoid/treat PFP development and its putative progression to knee osteoarthritis. This manuscript explores in detail each point involved in the formulation of the hypothesis. Additionally, directions for future research and possible clinical implications are discussed.

\section{Patellofemoral pain prognosis}

In a longitudinal study of 504 adolescents (ages 15-19) with knee pain, Rathleff et al. [18] reported that $55 \%$ of them continued to report knee pain two years later. Moreover, adolescents diagnosed with PFP had a greater probability to present pain after 2-years than those adolescents with knee pain due to other reasons [18]. In addition, $71 \%$ of adolescents with PFP reduced or stopped completely participation in sports activities after two years and more than a half of them reported knee pain daily or several times during the week [18]. Therefore, these findings demonstrate that PFP has a high potential for chronicity and reduced overall quality of life.

Even after treatment, individuals with PFP have an unfavorable prognosis [14,19]. Collins et al. [19] reported that $40 \%$ of 310 individuals with PFP who received different types of treatment classified their overall recovery between "moderate improvement" and "worse than ever" one year after the treatment [19]. That is, almost half of the individuals did not achieve symptom resolution 1-year after treatment. Following the same cohort of 310 individuals for about 5-8 years, it was observed that more than $50 \%$ continued to experience symptoms of PFP [14].

These studies highlight the need for investigating preventive strategies to reduce the proportion of individuals with PFP who develop severe long-term pain $[14,18,19]$, a possible predisposition to knee osteoarthritis [20]. Hence, risk factors associated with PFP need to be managed and not just the symptoms of the disorder. In this direction, one of the main reasons for the inefficacy of PFP treatments [19] is that the etiology of PFP remains unknown [21]. Currently, in addition to biomechanical, anatomical and muscle alterations, the association between neurophysiological changes and the presence of PFP has been cross-sectionally investigated $[16,17]$, but not in research designs that would investigate if they are risk factors.

\section{Progression of PFP to knee osteoarthritis}

Knee osteoarthritis is a degenerative disease, related to loss of joint cartilage and synovial inflammation, leading to stiffness, swelling, pain and loss of joint mobility [22]. Woolf and Pfleger [23] estimated that by 2020 , osteoarthritis will be the fourth largest cause of functional disability in adults and elderly, considering that in 1990 (just 30 years earlier) osteoarthritis occupied the eighth position [23]. In addition, in 2011, nearly 500,000 total knee arthroplasties were performed as treatment of knee osteoarthritis in the United States, and it is estimated the number of such surgical procedures will increase to 3.5 million by 2030 [9]. These data emphasize the importance of developing preventive and curative strategies to reduce public costs with osteoarthritis management. If PFP is indeed an osteoarthritis precursor, as recently suggested [6], proper management of PFP would act directly on the prevention of knee osteoarthritis, which grows exponentially in number of cases [9,23].

The putative continuity/progression of PFP to osteoarthritis was firstly investigated by Utting et al. [20]. In their study, $22 \%$ of the patients undergoing knee arthroplasty due to knee osteoarthritis in the PFJ reported chronic anterior knee pain which began in young adulthood (18 years) [20]. However, only $6 \%$ of the patients who underwent surgical treatment for tibiofemoral osteoarthritis reported previous anterior knee pain, which was also of shorter duration, less than 10 years [20]. Moreover, a recent systematic review [24] reported that $39 \%$ of individuals with knee pain had radiological signs compatible with presence of osteoarthritis in the PFJ [24]. Individuals with moderate osteoarthritis in PFJ show higher levels of pain and functional limitations when compared to those with moderate tibiofemoral osteoarthritis [25].

The possibility of osteoarthritis as PFP progression was discussed during the third and fourth Patellofemoral Pain Research Retreats $[3,21]$. Due to the short time from these meetings (2013-2015) until today, there is no prospective evidence of this progression. However, authorities in the PFP area have suggested that some similarities between individuals with PFP and PFJ osteoarthritis [6]. Crossley [6] reported that both PFP and PFJ osteoarthritis are associated with poor patellar alignment, quadriceps and hip abductors dysfunctions, difficulty in negotiating stairs and similar location of pain [6]. This was supported by a recent narrative review, in which Wyndow et al. [7] highlighted biomechanical changes associated with both conditions, such as patellar instability, femoral quadriceps muscle dysfunction and atrophy, dynamic knee misalignment, among others [7].

Therefore, if PFP is a risk factor or a sign of patellofemoral osteoarthritis onset, a condition that generates costs for public systems and health services around the world [6], then PFP should be the subject of more research and funding. According to this evidence, it is necessary to investigate and try to identify the risk factors for PFP in order to propose efficient treatment strategies to reverse the unfavorable prognosis of PFP [14,19].

\section{Patellofemoral pain and H-reflex modulation}

As PFP is a multifactorial musculoskeletal disorder, there are several biomechanical alterations associated with this condition. Factors that are associated with PFP include strength deficits in hip and quadriceps [26], and delayed VM activation among others [27]. Aminaka et al. [28] reported shorter time of VM activation in individuals with PFP as compared to pain-free controls during stair negotiation. The authors [28] raised the hypothesis that afferent sensory inputs from an injured joint can generate altered motor responses due to inhibitory interneuron activation that acts on the motor efferent pathway leading to a decrease in muscle activation [28]. This decreased muscle activation or muscle inhibition may be associated with peripheral, spinal and supraspinal mechanisms. In this direction, a recent systematic review [29] showed lower quadriceps central activation ratio in individuals with anterior knee pain as compared to individuals with anterior cruciate ligament injury, demonstrating that volitional quadriceps activation deficits are likely associated with anterior knee pain. Another study that used Magnetic Stimulation Mapping [15] showed that individuals with PFP presented reduction in map volume and a more anterior location of the representation of quadriceps muscles, which might be associated with reduced quadriceps corticomotor excitability in individuals PFP. However, no study has directly explored whether specific neurophysiological mechanisms might be considered risk factors for the development of PFP.

In this direction, the Hoffmann reflex, or H-reflex is a non-invasive experimental technique which can provide relevant information about neurophysiological mechanisms that regulate the excitability of spinal motoneurons [30]. The H-reflex was first described by Paul Hoffmann in 1910 [31], it is considered equivalent to the monosynaptic or stretching reflex generated by electrical stimulation and can be obtained in different muscles such as soleus, radial flexor of the carpus and quadriceps femoris [32]. In a study using this technique, Park and Hopkins [33] identified a $12 \%$ reduction in the H-reflex peak of painfree individuals after pain induction by sterile hypertonic saline solution into the infrapatellar fat pad [33]. Their findings suggest that the presence of pain may be one of the factors responsible for the inhibition of reflex pathway excitability. However, this result cannot be extrapolated to individuals with PFP, as there are particularities of PFP symptoms that should be taken into account, such as the fact that symptoms persist for long periods [14], intermittent pain [34], and 
diffuse pain location, ranging from the anterior knee, areas around the patella until medial areas of the thigh, being highly individual [35].

Taking into account these features of individuals with PFP and with evidence suggesting pain could be related to muscle inhibition, our research group began a new line of research $[16,17]$, thinking beyond proximal, local and distal biomechanical factors that have been widely studied in PFP research $[13,21,36]$. In order to explore the neurophysiological mechanisms associated with PFP, we reported that women with PFP presented lower VM H-reflex amplitude compared to pain-free controls [16]. Given these results, a subsequent study demonstrated that reduced H-reflex amplitude of the VM is associated with higher pain level, lower self-reported physical function and greater chronicity of pain in women with PFP. Therefore, it was found that women who experience PFP symptoms for a longer time also have reduced excitability of the VM H-reflex [17]. However, due to the cross-sectional design of the study, we could not explore any cause/effect relationship.

\section{Hypotheses}

There are no prospective studies investigating the relationship between lower H-reflex excitability and the development of PFP. Based on the identification of lower VM H-reflex excitability in women with PFP [16] and the strong association between H-reflex amplitude and chronicity of PFP [17], two hypotheses are plausible: (1) the reduction in excitability of the $\alpha$-motoneurons is a risk factor for PFP; Or, (2) the reduction in H-reflex excitability is a long-term consequence of pain. We propose a novel study where we measure the excitability of the $\mathrm{H}$ reflex at baseline in a large number of healthy women and follow them longitudinally for the appearance of the first symptoms of PFP. Besides H-reflex measurements, a complete assessment of the underlying neurophysiological mechanisms (including peripheral, spinal and supraspinal evaluations) might provide a more complete overview of the putative mechanisms associated with the development of PFP.

\section{Evaluation of the hypotheses}

In order to explore the hypotheses raised above we will evaluate if baseline H-reflex is a risk factor for the development of PFP. We intend to follow longitudinally the excitability of the H-reflex and the appearance of the first symptoms of PFP in a cohort of pain-free women. Additionally, on a smaller sample of the cohort, VM H-reflex excitability will be assessed monthly for six months, to obtain important information about the long-term reliability of VM H-reflex measurements.

\section{Clinical implication}

Clinically, it would be important to identify which hypothesis will be confirmed. If the H-reflex is a risk factor for PFP, the implementation of strategies to increase the excitability of the monosynaptic reflex pathway is necessary, once the alteration could be identified even without the presence of all PFP symptoms. Therefore, some interventions could be used as a prevention to avoid PFP development as well as its progression to knee osteoarthritis. On the other hand, if the H-reflex is a consequence of PFP, it is still necessary to manage it, in order to improve the prognosis of PFP and to increase the efficiency of PFP treatment in long-term, due to its association with higher pain level, lower self-reported physical function and greater chronicity of pain [17].

Such preventive or rehabilitation interventions will only be possible if the H-reflex is modifiable. It has been recently demonstrated that the operant conditioning of spinal reflex can modify the activity of spinal cord pathways, affecting the associated motor behavior [37]. In addition, a recent systematic review [38] has shown that transcutaneous electrical nerve stimulation is an effective way to increase voluntary activation of the quadriceps muscle. Thus, transcutaneous electrical nerve stimulation treatments may lead to an increase of VM H-reflex in women with PFP. Moreover, interventions like cryotherapy [39], proprioceptive exercises [40] and sensory stimulation [41] might be able to increase the excitation of the spinal cord by increasing the availability of descending and/or peripheral inputs, which may provide the best avenue to regain reflex excitability [42]. These strategies could be considered in combination with well-established evidence-based interventions for women with PFP [43].

\section{Conflict of interest statement}

The authors wish to confirm that there are no known conflicts of interest associated with this publication.

\section{References}

[1] Collaborators GB of DS. Global, regional, and national incidence, prevalence, and years lived with disability for 301 acute and chronic diseases and injuries in 188 countries, 1990-2013: a systematic analysis for the Global Burden of Disease Study 2013. Lancet 2013;2015(386):743-800. http://dx.doi.org/10.1016/S01406736(15)60692-4.

[2] Kannus P, Aho H, Järvinen M, et al. Computerized recording of visits to an outpatient sports clinic. Am J Sports Med 1987;15:79-85.

[3] Crossley KM, van Middelkoop M, Callaghan MJ, et al. 2016 Patellofemoral pain consensus statement from the 4th International Patellofemoral Pain Research Retreat, Manchester. Part 1: terminology, definitions, clinical examination, natural history, patellofemoral osteoarthritis and patient-reported outcome m. Br J Sports Med 2016;50:839-43. http://dx.doi.org/10.1136/bjsports-2016-096268.

[4] Roush JR, Bay RC. Prevalence of anterior knee pain in 18-35 year-old females. Int J Sports Phys Ther 2012;7:396-401.

[5] Boling M, Padua D, Marshall S, et al. Gender differences in the incidence and prevalence of patellofemoral pain syndrome. Scand J Med Sci Sports 2010;20:725-30. http://dx.doi.org/10.1111/j.1600-0838.2009.00996.x.

[6] Crossley KM. Is patellofemoral osteoarthritis a common sequela of patellofemoral pain? Br J Sports Med 2014;48:409-10. http://dx.doi.org/10.1136/bjsports-2014093445.

[7] Wyndow N, Collins N, Vicenzino B, et al. Is there a biomechanical link between patellofemoral pain and osteoarthritis? A narrative review. Sport Med 2016:46:1797-808. http://dx doi org/10.1007/s40279-016-0545-6.

[8] Pereira D, Peleteiro B, Araújo J, et al. The effect of osteoarthritis definition on prevalence and incidence estimates: a systematic review. Osteoarthr Cartil 2011;19:1270-85. http://dx.doi.org/10.1016/j.joca.2011.08.009.

[9] Kurtz S, Ong K, Lau E, et al. Projections of primary and revision hip and knee arthroplasty in the United States from 2005 to 2030. J Bone Jt Surg Am 2007;89:780-5.

[10] De Oliveira Silva D, Barton CJ, Pazzinatto MF, et al. Proximal mechanics during stair ascent are more discriminate of females with patellofemoral pain than distal mechanics. Clin Biomech 2016;35:56-61. http://dx.doi.org/10.1016/j. clinbiomech.2016.04.009.

[11] De Oliveira Silva D, Briani RV, Pazzinatto MF, et al. Reduced knee flexion is a possible cause of increased loading rates in individuals with patellofemoral pain. Clin Biomech 2015;30:971-5. http://dx.doi.org/10.1016/j.clinbiomech.2015.06. 021.

[12] De Oliveira Silva D, Magalhães FH, Pazzinatto MF, et al. Contribution of altered hip, knee and foot kinematics to dynamic postural impairments in females with patellofemoral pain during stair ascent. Knee 2016;23:376-81. http://dx.doi.org/10. 1016/j.knee.2016.01.014.

[13] Powers CM, Bolgla La, Callaghan MJ, et al. Patellofemoral pain: proximal, distal, and local factors, 2nd International Research Retreat. J Orthop Sports Phys Ther 2012;42:A1-54. http://dx.doi.org/10.2519/jospt.2012.0301.

[14] Lankhorst NE, van Middelkoop M, Crossley KM, et al. Factors that predict a poor outcome 5-8 years after the diagnosis of patellofemoral pain: a multicentre observational analysis. Br J Sports Med 2016;50:881-6. http://dx.doi.org/10.1136/ bjsports-2015-094664.

[15] Te M, Baptista AF, Chipchase LS, et al. Primary motor cortex organization is altered in persistent patellofemoral pain. Pain Med 2017:1-11. http://dx.doi.org/10.1093/ $\mathrm{pm} / \mathrm{pnx} 036$.

[16] De Oliveira Silva D, Magalhães FH, Faria NC, et al. Lower amplitude of the hoffmann reflex in women with patellofemoral pain: thinking beyond proximal, local, and distal factors. Arch Phys Med Rehabil 2016;97:1115-20. http://dx.doi.org/10 1016/j.apmr.2015.12.017.

[17] De Oliveira Silva D, Magalhães FH, Faria NC, et al. Vastus medialis H-reflex excitability is associated with pain level, self-reported function and chronicity in women with patellofemoral pain. Arch Phys Med Rehabil 2017;98:114-9. http:// dx.doi.org/10.1016/j.apmr.2016.06.011.

[18] Rathleff MS, Rathleff CR, Olesen JL, et al. Is knee pain during adolescence a selflimiting condition? Prognosis of patellofemoral pain and other types of knee pain Am J Sports Med 2016;44:1165-71. http://dx.doi.org/10.1177/ 0363546515622456.

[19] Collins NJ, Bierma-Zeinstra SMa, Crossley KM, et al. Prognostic factors for patellofemoral pain: a multicentre observational analysis. Br J Sports Med 
2013;47:227-33. http://dx.doi.org/10.1136/bjsports-2012-091696.

[20] Utting MR, Davies G, Newman JH. Is anterior knee pain a predisposing factor to patellofemoral osteoarthritis? Knee 2005;12:362-5. http://dx.doi.org/10.1016/j. knee.2004.12.006.

[21] Witvrouw E, Callaghan MJ, Stefanik JJ, et al. Patellofemoral pain: consensus statement from the 3rd International Patellofemoral Pain Research Retreat held in Vancouver, September 2013. Br J Sports Med 2014;48:411-4. http://dx.doi.org/10. 1136/bjsports-2014-093450.

[22] Musumeci G, Aiello F, Szychlinska M, et al. Osteoarthritis in the XXIst century: risk factors and behaviours that influence disease onset and progression. Int $\mathrm{J}$ Mol Sci 2015;16:6093-112. http://dx.doi.org/10.3390/ijms16036093.

[23] Woolf AD, Pfleger B. Burden of major musculoskeletal conditions. Bull World Health Organ 2003;81:646-56. S0042-96862003000900007 [pii].

[24] Kobayashi S, Pappas E, Fransen M, et al. The prevalence of patellofemoral osteoarthritis: a systematic review and meta-analysis. Osteoarthr Cartil 2016;24:1697-707. http://dx.doi.org/10.1016/j.joca.2015.02.968.

[25] Duncan R, Peat G, Thomas E, et al. How do pain and function vary with compartmental distribution and severity of radiographic knee osteoarthritis? Rheumatology 2008;47:1704-7. http://dx.doi.org/10.1093/rheumatology/ ken339.

[26] Lankhorst NE, Bierma-Zeinstra SM, van Middelkoop M. Risk factors for patellofemoral pain syndrome: a systematic review. J Orthop Sports Phys Ther 2012;42:81-94. http://dx.doi.org/10.2519/jospt.2012.3803.

[27] Uliam Kuriki H, Mícolis de Azevedo F, de Faria Negrão Filho R, et al. Comparison of different analysis techniques for the determination of muscle onset in individuals with patellofemoral pain syndrome. J Electromyogr Kinesiol 2011;21:982-7. http://dx.doi.org/10.1016/j.jelekin.2011.08.002.

[28] Aminaka N, Pietrosimone BG, Armstrong CW, et al. Patellofemoral pain syndrome alters neuromuscular control and kinetics during stair ambulation. J Electromyogr Kinesiol 2011;21:645-51. http://dx.doi.org/10.1016/j.jelekin.2011.03.007.

[29] Hart JM, Pietrosimone B, Hertel J, et al. Quadriceps activation following knee injuries: a systematic review. J Athl Train 2010;45:87-97. http://dx.doi.org/10. 4085/1062-6050-45.1.87.

[30] Pierrot-Deseilligny E, Burke D. The circuitry of the human spinal cord: its role in motor control and movement disorders. New York: Cambridge University Press; 2005.

[31] Hoffmann P. Beitrag zur Kenntnis der menschlichen Reflexe mit besonderer Berucksichtigung der elektrischen Erscheinungen. Arch Anat Physiol 1910;1:223-46.

[32] Burke D. Clinical uses of $\mathrm{H}$ reflexes of upper and lower limb muscles. Clin
Neurophysiol Pract 2016;1:9-17. http://dx.doi.org/10.1016/j.cnp.2016.02.003.

[33] Park J, Hopkins JT. Induced anterior knee pain immediately reduces involuntary and voluntary quadriceps activation. Clin J Sport Med 2013;23:19-24. http://dx. doi.org/10.1097/JSM.0b013e3182717b7b.

[34] Pazzinatto MF, De Oliveira Silva D, Barton C, et al. Female adults with patellofemoral pain are characterized by widespread hyperalgesia, which is not affected immediately by patellofemoral joint loading. Pain Med 2016;17:1953-61. http:// dx.doi.org/10.1093/pm/pnw068.

[35] Rathleff MS, Petersen KK, Arendt-Nielsen L, et al. Impaired conditioned pain modulation in young female adults with long-standing patellofemoral pain: a single blinded cross-sectional study. Pain Med 2016;17:980-8. http://dx.doi.org/10. 1093/pm/pnv017.

[36] Davis IS, Powers CM. Patellofemoral pain syndrome: proximal, distal, and local factors - An International Research Retreat. J Orthop Sports Phys Ther 2010;40:A1-48. http://dx.doi.org/10.2519/jospt.2010.

[37] Thompson AK, Wolpaw JR. The simplest motor skill: mechanisms and applications of reflex operant conditioning. Exerc Sport Sci Rev 2014;42:82-90. http://dx.doi. org/10.1249/JES.0000000000000010.

[38] Harkey MS, Gribble PA, Pietrosimone BG. Disinhibitory interventions and voluntary quadriceps activation: a systematic review. J Athl Train 2014;49:411-21. http://dx. doi.org/10.4085/1062-6050-49.1.04.

[39] Hart JM, Kuenze CM, Diduch DR, et al. Quadriceps muscle function after rehabilitation with cryotherapy in patients with anterior cruciate ligament reconstruction. J Athl Train 2014;49:733-9. http://dx.doi.org/10.4085/1062-605049.3.39.

[40] Taube W, Kullmann N, Leukel C, et al. Differential reflex adaptations following sensorimotor and strength training in young elite athletes. Int J Sports Med 2007;28:999-1005. http://dx.doi.org/10.1055/s-2007-964996.

[41] Perez MA, Field-Fote EC, Floeter MK. Patterned sensory stimulation induces plasticity in reciprocal ia inhibition in humans. J Neurosci 2003;23:2014-8. 23/6/2014 [pii].

[42] Motta-Oishi AAP, Magalhães FH, Mícolis de Azevedo F. Neuromuscular electrical stimulation for stroke rehabilitation: is spinal plasticity a possible mechanism associated with diminished spasticity? Med Hypotheses 2013;81:784-8. http://dx. doi.org/10.1016/j.mehy.2013.08.013.

[43] Barton CJ, Lack S, Hemmings S, et al. The 'Best Practice Guide to Conservative Management of Patellofemoral Pain': incorporating level 1 evidence with expert clinical reasoning. Br J Sports Med 2015;49:923-34. http://dx.doi.org/10.1136/ bjsports-2014-093637. 